\title{
Clinical validation of a blood-based classifier for diagnostic evaluation of asymptomatic individuals with pulmonary nodules
}

\author{
Charles E. Birse ${ }^{1 *} \mathbb{D}$, Jennifer L. Tomic ${ }^{1,4}$, Harvey I. Pass ${ }^{2}$, William N. Rom³ and Robert J. Lagier ${ }^{1}$
}

\begin{abstract}
Background: The number of pulmonary nodules detected in the US is expected to increase substantially following recent recommendations for nationwide CT-based lung cancer screening. Given the low specificity of CT screening, non-invasive adjuvant methods are needed to differentiate cancerous lesions from benign nodules to help avoid unnecessary invasive procedures in the asymptomatic population. We have constructed a serum-based multi-biomarker panel and assessed its clinical accuracy in a retrospective analysis of samples collected from participants with suspicious radiographic findings in the Prostate, Lung, Chest and Ovarian (PLCO) cancer screening trial.
\end{abstract}

Methods: Starting with a set of 9 candidate biomarkers, we identified 8 that exhibited limited pre-analytical variability with increasing clotting time, a key pre-analytical variable associated with the collection of serum. These 8 biomarkers were evaluated in a training study consisting of 95 stage I NSCLC patients and 186 smoker controls where a 5-biomarker pulmonary nodule classifier (PNC) was selected. The clinical accuracy of the PNC was determined in a blinded study of asymptomatic individuals comprising 119 confirmed malignant nodule cases and 119 benign nodule controls selected from the PLCO screening trial.

Results: A PNC comprising 5 biomarkers: CEA, CYFRA 21-1, OPN, SCC, and TFPI, was selected in the training study. In an independent validation study, the PNC resolved lung cancer cases from benign nodule controls with an AUC of 0.653 ( $p<0.0001)$. CEA and CYFRA 21-1, two of the markers included in the PNC, also accurately distinguished malignant lesions from benign controls.

Conclusions: A 5-biomarker blood test has been developed for the diagnostic evaluation of asymptomatic individuals with solitary pulmonary nodules.

Keywords: Lung cancer screening, Indeterminate pulmonary nodules, Biomarker, Serum, Asymptomatic

\section{Background}

Pulmonary nodules are a common finding in routine clinical practice with an estimated 1.5 million new cases being detected annually in the United States [1]. Incidence is likely to increase in the coming years following the recommendations from the US Preventative Services

\footnotetext{
*Correspondence: Charles.E.Birse@questdiagnostics.com

${ }^{1}$ Quest Diagnostics, Research and Development, 33608 Ortega Highway, San Juan Capistrano, CA 92675, USA

Full list of author information is available at the end of the article
}

Task Force (USPSTF) [2] and the Centers for Medicare \& Medicaid Services (CMS) [3] for annual CT-based screening of the population with a high risk for lung cancer. Currently there are approximately 7 million current or former smokers in the US who meet the National Lung Screening Trial (NLST) criteria for screening. In the NLST, $24.2 \%$ of CT screening tests had a positive finding, with $96.4 \%$ of these initial findings representing false positives for lung cancer [4].

Given the poor prognosis associated with advanced stage disease, the goal in managing individuals with 
pulmonary nodules is to rapidly identify and resect malignant lesions while avoiding unnecessary invasive procedures in patients with benign disease. Guidelines for the management of pulmonary nodules have recently been revised by several professional organizations [5-7]. The American College of Radiology has initiated efforts to standardize reporting of results from low dose chest CT screening with the introduction of the Lung-RADS system, which emulates the Bi-RADS scoring system for mammography. Lung-RADS, which defines a positive screening test and provides recommendations for management based on level of risk, has been adopted at many academic medical centers in the US [8]. The Lung-RADS system increases the size threshold for a positive baseline finding from $4 \mathrm{~mm}$, used in the NLST, to $6 \mathrm{~mm}$. $\geq 6$ to $<8 \mathrm{~mm}$ nodules (Category 3) are considered "probably benign", with a risk of malignancy of $1-2 \%$ and should be managed with a follow-up low dose CT scan in 6 months. Category 4 nodules are considered "suspicious": $\geq 8$ to $<15 \mathrm{~mm}$ nodules (Category 4A) have a $5-15 \%$ cancer risk and should be managed with a 3 month LDCT scan, or $\mathrm{PET} / \mathrm{CT}$ may be used if there is a $\geq 8 \mathrm{~mm}$ solid component; $>15 \mathrm{~mm}$ nodules (Category $4 \mathrm{~B}$ ) have a cancer risk $\geq 15 \%$ and should be managed with a chest CT with/ without contrast, PET/CT and/or biopsy, depending on the risk of malignancy and comorbidities. Despite these updated recommendations, invasive procedures are still performed on $44 \%$ of subjects with low-risk nodules $(<5 \%$ probability of malignancy), and $35 \%$ of surgical resections are ultimately determined to be benign disease [9].

Integration of blood-based biomarkers may increase the accuracy of nodule classification, improving diagnostic evaluation, and thereby reducing the number of unnecessary and costly invasive procedures [10]. Although some promising candidate protein molecules have been evaluated as single markers [11, 12], given the heterogeneity of non-small cell lung cancers, it seems likely that multi-marker panels will be required to provide sufficient test sensitivity to make a meaningful clinical impact in nodule management. Some multi-marker adjunctive tests have been shown to perform with modest diagnostic accuracy in distinguishing benign from malignant lesions in subjects with indeterminate pulmonary nodules [13-18].

Given the recent recommendations from USPSTF and CMS for CT-based screening of those at high risk of lung cancer, there is a pressing need to investigate the performance of proteomic biomarkers in this asymptomatic population. While a number of well characterized markers including CEA, CYFRA 21-1 and SCC have previously been assessed for a wide range of diagnostic, prognostic and monitoring applications, they have not been evaluated as adjunctive biomarkers in the radiographic screening of asymptomatic individuals for lung cancer [19-22]. Some investigations have assessed the performance of these markers in individuals with pulmonary nodules, but these studies, including earlier work in our laboratory [23], have included symptomatic lung cancer cases, or controls not restricted to subjects with benign nodules $[13,14,24]$.

We previously identified a diverse repertoire of candidate lung cancer biomarkers using a mass spectrometrybased discovery approach. We now report on the transfer of these markers onto a multiplex Luminex platform, thereby increasing throughput and reducing sample volume requirements. Using this multiplex assay, we deselected biomarkers affected by pre-analytical variables associated with blood collection and processing [25-28]. Thereafter, we developed a serum-based pulmonary nodule classifier (PNC) comprising 5 biomarkers: CEA, CYFRA 21-1, OPN, SCC, and TFPI. We then determined the clinical accuracy of the PNC, together with the performance of the individual contributing biomarkers, in asymptomatic subjects with suspicious radiographic abnormalities from the Prostate, Lung, Chest and Ovarian (PLCO) cancer screening trial [29]. We believe this study provides the first evaluation of clinical accuracy of these biomarkers in individuals with solitary pulmonary nodules resolved through radiographic screening for lung cancer.

\section{Methods}

\section{Pre-analytical variability study}

Blood was drawn from healthy volunteers $(n=6)$. From each individual, 3 blood samples were collected into redtop serum tubes (Becton Dickinson, Franklin Lakes, NJ, \#367820) and blood was allowed to clot at room temperature for: $0.5,4$, or $24 \mathrm{~h}$. After clotting, tubes were spun at $1200 \mathrm{~g}$ for $10 \mathrm{~min}$ at room temperature. $2 \mathrm{~mL}$ aliquots of separated serum were then transferred to $-80{ }^{\circ} \mathrm{C}$ for long-term storage.

\section{Training study (New York University, Clinical Research Center at Cape Cod)}

Serum was collected from healthy smoker controls $(\mathrm{n}=186)$ and patients with early-stage non-small-cell lung cancer (NSCLC; $\mathrm{n}=95$ ) (Table 1 ).

The design and subjects evaluated, are very similar to a previous training study where biomarker levels were evaluated using an ELISA platform [23].

\section{Validation study (PLCO)}

PLCO biospecimens were collected and processed using uniform procedures. Samples were collected prospectively (pre-diagnosis), eliminating any inherent case-control bias [30]. 
Table 1 Demographics of the training and validation study populations

\begin{tabular}{|c|c|c|c|c|}
\hline & \multicolumn{2}{|l|}{ Training } & \multirow{2}{*}{\multicolumn{2}{|c|}{$\frac{\text { Validation }}{\text { CXR finding: nodule }}$}} \\
\hline & \multirow{2}{*}{$\begin{array}{l}\text { Control } \\
(n=186)\end{array}$} & \multirow{2}{*}{$\begin{array}{l}\text { NSCLC } \\
(n=95)\end{array}$} & & \\
\hline & & & $\begin{array}{l}\text { Control } \\
(n=119)\end{array}$ & $\begin{array}{l}\text { Lung cancer } \\
(\mathrm{n}=119)\end{array}$ \\
\hline \multicolumn{5}{|l|}{ Gender } \\
\hline Male & 107 & 34 & 69 & 69 \\
\hline Female & 79 & 61 & 50 & 50 \\
\hline \multicolumn{5}{|l|}{ Age (years) } \\
\hline Mean (SD) & $62.3(11.8)$ & $66.5(9.6)$ & $63.7(5.2)$ & $64.4(5.3)$ \\
\hline \multicolumn{5}{|l|}{ Smoking } \\
\hline $\begin{array}{l}\text { Ever smok- } \\
\text { ers }\end{array}$ & 186 & 95 & 106 & 106 \\
\hline $\begin{array}{l}\text { Never } \\
\text { smokers }\end{array}$ & - & - & 13 & 13 \\
\hline $\begin{array}{l}\text { Pack years, } \\
\text { mean } \\
\text { (SD) }\end{array}$ & $37.4(21.5)$ & $44.5(21.3)$ & $46.0(32.4)$ & $55.3(40.9)$ \\
\hline \multicolumn{5}{|c|}{ Abnormal suspicious finding } \\
\hline Nodule & NA & NA & 119 & 119 \\
\hline \multicolumn{5}{|l|}{ Stage } \\
\hline 1 & & $95(100 \%)$ & & $5(4.2 \%)$ \\
\hline la & & - & & $51(42.9 \%)$ \\
\hline $\mathrm{lb}$ & & - & & $15(12.6 \%)$ \\
\hline$\|$ & & - & & $10(8.4 \%)$ \\
\hline III & & - & & $19(16.0 \%)$ \\
\hline IV & & - & & $14(11.8 \%)$ \\
\hline NA & & - & & $5(4.2 \%)$ \\
\hline \multicolumn{5}{|l|}{ Histology } \\
\hline $\begin{array}{c}\text { Adenocar- } \\
\text { cinoma }\end{array}$ & & $63(66.3 \%)$ & & $57(47.9 \%)$ \\
\hline BAC & & $4(4.2 \%)$ & & $20(16.8 \%)$ \\
\hline $\begin{array}{l}\text { Squamous } \\
\text { cell }\end{array}$ & & $15(15.8 \%)$ & & $25(21.0 \%)$ \\
\hline Large cell & & $6(6.3 \%)$ & & $4(3.4 \%)$ \\
\hline NSCLC & & $4(4.2 \%)$ & & $2(1.7 \%)$ \\
\hline $\begin{array}{l}\text { Neuroen- } \\
\text { docrine }\end{array}$ & & $2(2.1 \%)$ & & $2(1.7 \%)$ \\
\hline Small cell & & - & & $5(4.2 \%)$ \\
\hline $\begin{array}{l}\text { Adenos- } \\
\text { quamous }\end{array}$ & & $1(1.1 \%)$ & & $4(3.4 \%)$ \\
\hline
\end{tabular}

In the intervention (screening) arm of the PLCO study, 77,445 participants received a chest radiograph at baseline, then annually for 2 or 3 years [31]. Chest radiographs were considered positive if a nodule, mass, or other suspicious abnormality (atelectasis, pleural, hilar or mediastinal mass) was found. The prevalence of screen-detect cancers was strongly influenced by the type of abnormality. The PPV for a nodule was $1.3 \%$ $(170 / 13,449)$, for a mass $8.7 \%(105 / 1208)$, and for other suspicious abnormalities 1.9\% (63/3356) [32,33]. Cancers were staged based on the fifth edition of the American Joint Committee on Cancer Staging Manual [34].

Subjects with solitary abnormal suspicious findings (nodule, mass, other) were considered for our validation study. Cases selected included individuals with a nodule $(<3 \mathrm{~cm} ; \mathrm{n}=119)$, a mass $(>3 \mathrm{~cm} ; \mathrm{n}=50)$, or "other" findings (pleural mass, hilar, infiltrate; $\mathrm{n}=28$ ), for whom serum had been collected up to 12 months before the diagnosis of malignancy. Controls were required to have at least 2 years of follow-up. Controls were individually matched to cases based on: finding (nodule, mass, other), gender, age at randomization and smoking history (Tables 1, 2). 152 of the 222 cases $(68 \%)$ included in the study met the NLST high risk criteria where participants were selected based on age (55-74 years) and smoking history (at least $30+$ pack years).

\section{Multiplex bead-based immunoassay}

$\mathrm{xMAP}^{\mathrm{TM}}$ bead-based technology (Luminex Corp., Austin, TX) enables simultaneous analysis of multiple analytes in a single sample. To ensure measurement of linear responses for all 9 candidate lung cancer biomarkers over a broad dynamic range, 5 of the biomarkers (MMP2, OPN, SLPI, TFPI, and TIMP1) were measured in serum diluted ten-fold in assay buffer (\#RD-48; R\&D Systems/ Bio-Techne, Minneapolis, MN). Levels of the 4 remaining biomarkers (CYFRA 21-1, CEA, SCC and MDK) were measured in undiluted serum. Biomarker-specific reagents employed in these studies are described in Additional file 1: Table S1. Primary antibodies were coupled to magnetic carboxylated beads (MagPlex ${ }^{\circledR}$ Microspheres, Luminex Corp.), following the manufacturer's procedure, with the addition of an ethanolamine quenching step prior to blocking. Secondary antibodies were labeled through EZ-Link Sulfo-NHS-Biotinylation (Thermo Scientific Pierce, Grand Island, NY).

$\mathrm{xMAP}^{\mathrm{TM}}$ assays were performed in 96-well format following the manufacturer's protocol (Luminex Corp), with some modifications. $6 \mu \mathrm{L}$ of standards or serum were transferred in triplicate to the plate. Conjugated bead mixtures were sonicated briefly before being added $(24 \mu \mathrm{L})$ to each well. Plates were covered with foil tape, and shaken overnight at $4{ }^{\circ} \mathrm{C}$. Following transfer to a pre-wetted filter plate (AcroPrep 96; Pall Corp, Port Washington, NY), wells were washed 3 times (\#WA126; R\&D Systems/BioTechne) using a 406EL washer (BioTek, Winooski, VT). Biotin-labeled secondary antibody $(25 \mu \mathrm{L})$ was added to each well, and the plates were shaken at room temperature for $120 \mathrm{~min}$. After washing, $25 \mu \mathrm{L}$ of diluted StreptavidinPE (\# PJRS20; Prozyme, Hayward, CA) was added to each well, and the plate was shaken for $30 \mathrm{~min}$. After another wash step, beads were resuspended in $100 \mu \mathrm{L}$ of PBS 
Table 2 Demographics of the validation study populations (mass and other)

\begin{tabular}{|c|c|c|c|c|}
\hline & \multicolumn{4}{|l|}{ Validation } \\
\hline & \multicolumn{2}{|c|}{$\begin{array}{l}\text { CXR finding: mass } \\
(n=100)\end{array}$} & \multicolumn{2}{|c|}{$\begin{array}{l}\text { CXR finding: other } \\
(n=56)\end{array}$} \\
\hline & Control & Lung cancer & Control & Lung cancer \\
\hline \multicolumn{5}{|l|}{ Gender } \\
\hline Male & 28 & 28 & 22 & 22 \\
\hline Female & 22 & 22 & 6 & 6 \\
\hline \multicolumn{5}{|l|}{ Age (years) } \\
\hline Mean (SD) & $64.0(4.9)$ & $63.9(4.9)$ & $65.3(5.5)$ & $65.1(4.9)$ \\
\hline \multicolumn{5}{|l|}{ Smoking } \\
\hline Ever smokers & 45 & 45 & 28 & 28 \\
\hline Never smokers & 5 & 5 & - & - \\
\hline $\begin{array}{l}\text { Pack years mean } \\
\text { (SD) }\end{array}$ & $50.6(35.1)$ & $58.2(37.9)$ & $54.8(32.9)$ & $52.3(30.9)$ \\
\hline \multicolumn{5}{|c|}{ Abnormal suspicious finding } \\
\hline Mass & 50 & 50 & - & - \\
\hline Pleural Mass & - & - & 2 & 2 \\
\hline Hilar & - & - & 9 & 9 \\
\hline Infiltrate & - & - & 17 & 17 \\
\hline \multicolumn{5}{|l|}{ Stage } \\
\hline l (\%) & & $16(32.0)$ & & $9(32.1)$ \\
\hline II (\%) & & $4(8.0)$ & & $3(10.7)$ \\
\hline III (\%) & & $16(32.0)$ & & $3(10.7)$ \\
\hline IV (\%) & & $10(20.0)$ & & $8(28.6)$ \\
\hline NA & & $4(8.0)$ & & $5(17.9)$ \\
\hline \multicolumn{5}{|l|}{ Histology } \\
\hline $\begin{array}{c}\text { Adenocarci- } \\
\text { noma (\%) }\end{array}$ & & $24(48.0)$ & & $12(42.9)$ \\
\hline BAC (\%) & & $3(6.0)$ & & $2(7.1)$ \\
\hline $\begin{array}{l}\text { Squamous cell } \\
(\%)\end{array}$ & & $9(18.0)$ & & $7(25.0)$ \\
\hline Large cell (\%) & & $4(8.0)$ & & $1(3.6)$ \\
\hline NSCLC (\%) & & $6(12.0)$ & & $1(3.6)$ \\
\hline Small cell (\%) & & $4(8.0)$ & & $5(17.9)$ \\
\hline
\end{tabular}

before being read (Luminex 200). MasterPlex QT curvefitting software (Hitachi Solutions America, San Bruno, CA) was used to analyze Luminex $\mathrm{xMAP}^{\mathrm{TM}}$ data.

\section{Statistical methods}

The final set of biomarkers for the PNC was selected via elasticnet regularized logistic regression modeling of lung cancer status [35]. The elasticnet regularization (95\% lasso, $5 \%$ ridge) constrains the regression coefficients in an effort to increase prediction accuracy by controlling for overfitting of the model to the training data. Additionally, elasticnet performs marker selection by forcing the coefficients of the least contributory markers to shrink to zero as the penalty parameter is increased. Stratified bootstrap resampling
(10,000 iterations) was used to select the optimal regularization penalty. Subjects not selected in each bootstrap sample were scored as a test set by each penalized model. The regularization penalty which achieved the greatest mean AUC for the 10,000 test sets was selected for regularizing the final fit to the full training data set. We chose maximal mean AUC as the criterion for selection of the elasticnet penalty parameter as it is an accepted and commonly used measure of overall performance in the classification setting. Other measures such as NPV and PPV could have been used to select the penalty, but this would introduce a level of subjectivity into the method as one would need to determine appropriate levels of each of these performance measures without any clear guidelines for such decisions. Confidence intervals for AUCs and tests for differences in AUCs for paired samples were calculated by the DeLong [36] method. All statistical analyses were performed in R [37].

\section{Results}

Previous evaluation of the 9 candidate lung cancer biomarkers was performed using singleplex assays configured using ELISA methodology [23]. A new multiplex version of the assay was developed to streamline clinical validation studies using Luminex technology. The multiplex assay is highly specific with $<1 \%$ cross-reactivity among markers (Additional file 1: Table S2).

One of the key pre-analytical variables in serum collection is clotting time, the period from venipuncture to centrifugation of the blood sample, with investigators employing a diverse spectrum of clotting-times, ranging from 30-60 $\mathrm{min}$ [38] to $24-56 \mathrm{~h}$ [27]. We evaluated the influence of clotting time in order to deselect candidate biomarkers affected by this key step in the pre-analytical processing pathway. We investigated the effect of clotting time on the serum levels of the 9 candidate lung cancer biomarkers, determining levels in six healthy individuals after clot formation for: $0.5,4$ or $24 \mathrm{~h}$. The levels of most of the biomarkers showed only minor fluctuations at either 4 or $24 \mathrm{~h}$, relative to the $0.5 \mathrm{~h}$ time point. However, levels of MDK fell consistently at both the 4-h (mean decrease $26 \%$ ) and 24-h time points (mean decrease $48 \%$ ), with lower levels always observed after $24 \mathrm{~h}$ (Fig. 1). Therefore, we excluded this biomarker from further evaluation.

Multi-marker algorithms started with the 8 biomarkers that showed minimal levels of pre-analytical variability. A 5-biomarker model (CEA, CYFRA 21-1, OPN, SCC and TFPI), a pulmonary nodule classifier (PNC), was selected from the training dataset (Additional file 1: Table S3). The PNC resolved malignant cases from healthy smoker controls with an AUC of 0.897 (95\% CI 0.857-0.937; Additional file 2: Figure S1). A bootstrap validation procedure $(10,000$ iterations) confirmed the accuracy of the PNC in the training study: AUC was 0.894 (95\% CI 0.822-0.944). 


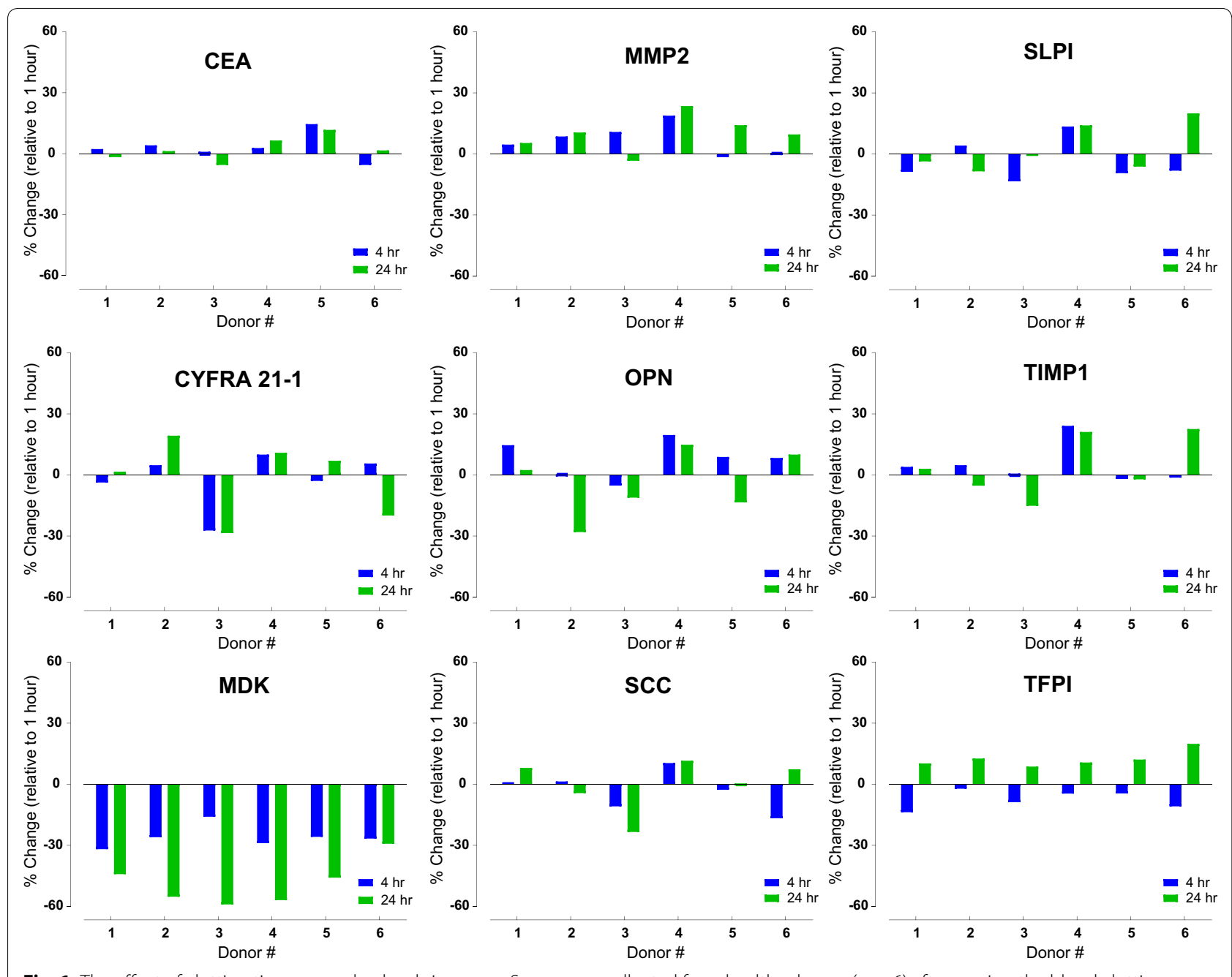

Fig. 1 The effect of clotting time on marker levels in serum. Serum was collected from healthy donors $(n=6)$ after varying the blood clotting period: $0.5,4,24 \mathrm{~h}$. Changes in marker levels observed after clotting for $4 \mathrm{~h}$ (blue bar) or $24 \mathrm{~h}$ (green bar) were plotted relative to the $0.5 \mathrm{~h}$ time point

The accuracy of the PNC in distinguishing individuals with benign pulmonary nodules $(\mathrm{n}=119)$ from those with malignant lesions $(\mathrm{n}=119)$ was tested in serum collected pre-diagnosis from individuals participating in the PLCO cancer screening trial (Table 1). The PNC resolved the 2 populations with an AUC of $0.653, \mathrm{p}<0.0001$ (Additional file 2: Figure S2). The performance of the individual biomarkers that constitute the classifier was also assessed, with CEA (AUC $=0.642, \mathrm{p}<0.0001$ ) and CYFRA 21-1 (AUC $=0.628, p=0.0004$ ) accurately distinguishing cases from controls (Table 3 ).

The accuracy of the PNC in patients with pulmonary nodules was further characterized relative to tumor stage and histology. The multi-biomarker test showed modest performance in resolving stage IA cases $(n=51)$, which comprise $43 \%$ of the malignant nodules (AUC $=0.618$, $\mathrm{p}=0.0071$; Fig. 2). Stage IA cases represent tumors $\leq 3 \mathrm{~cm}$ without bronchoscopic evidence of invasion more proximal than the lobar bronchus. Improved test accuracy was observed for cases diagnosed at later stages: stage II: AUC $=0.695(\mathrm{p}=0.0341)$, stage III: AUC $=0.766(p<0.0001)$ and stage IV: AUC $=0.742$ $(p=0.0009)$. Enhanced accuracy in resolving late stage disease (relative to stage I) was also noted for two biomarkers in the model: CEA and CYFRA 21-1 (Additional file 1: Table S4), as has previously been reported [19-22].

The PNC accurately classified the most prevalent NSCLC histological cell types: adenocarcinoma, AUC $=0.665(\mathrm{p}=0.0001)$ and squamous cell carcinoma, $\mathrm{AUC}=0.709(\mathrm{p}=0.0005)$ from benign controls, as shown in Fig. 3. Analysis of individual biomarkers revealed that CEA and CYFRA 21-1 also distinguished adenocarcinoma (CEA: AUC $=0.665, p=0.0004$; CYFRA 21-1: $\mathrm{AUC}=0.629, \mathrm{p}=0.0033$ ) and squamous cell histologies (CEA: AUC $=0.649, \mathrm{p}=0.0180$; CYFRA 21-1: $\mathrm{AUC}=0.643, \mathrm{p}=0.0484)$ as shown in Additional 
Table 3 Diagnostic accuracy of pulmonary nodule classifier (PNC) and individual markers evaluated in the nodule population of the validation study

\begin{tabular}{llr}
\hline & \multicolumn{1}{l}{ Validation } \\
\cline { 2 - 3 } & $\begin{array}{l}\text { CXR finding: nodule } \\
\text { Benign control }(\mathbf{n}=119) ; \\
\end{array}$ & \multicolumn{2}{l}{ Lung cancer $(\mathbf{n}=\mathbf{1 1 9})$} \\
\cline { 2 - 3 } & AUC [95\% Cl] & p value \\
\hline PNC & $0.653[0.583-0.723]$ & $<0.0001$ \\
Individual markers included in PNC & & \\
CEA & $0.642[0.572-0.713]$ & $<0.0001$ \\
CYFRA 21-1 & $0.628[0.558-0.699]$ & 0.0004 \\
SCC & $0.567[0.494-0.640]$ & 0.0737 \\
OPN & $0.535[0.461-0.609]$ & 0.3508 \\
TFPI & $0.533[0.459-0.606]$ & 0.3815 \\
\hline
\end{tabular}

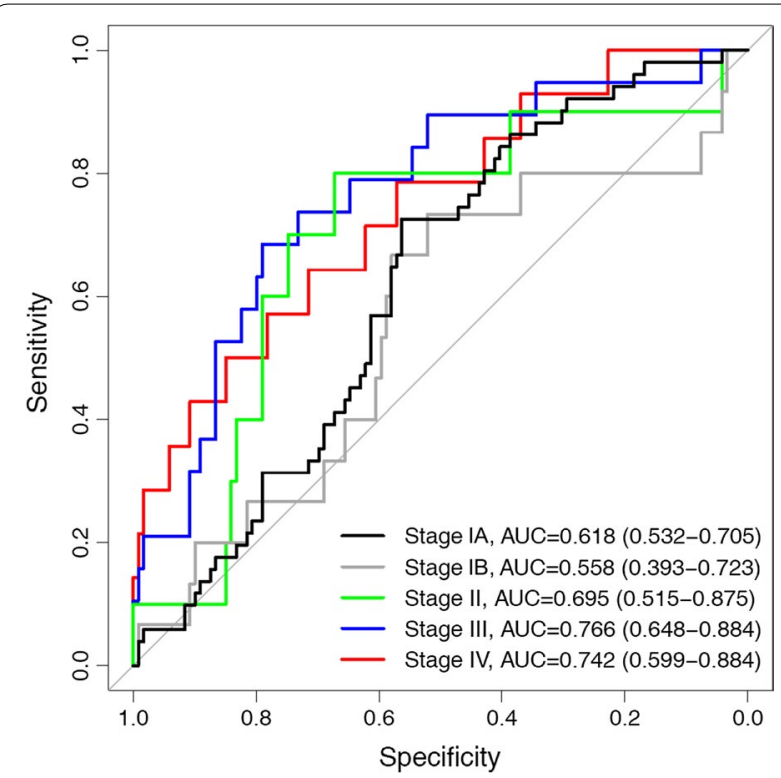

Fig. 2 ROC curves showing diagnostic accuracy of pulmonary nodule classifier (PNC) evaluated in PLCO nodule population relative to tumor stage: stage IA ( $n=51$, black), stage IB $(n=15$, grey), stage II ( $n=10$, green), stage III $(n=19$, blue) and stage IV $(n=13$, red). Area under the curve (AUC) and $95 \%$ confidence intervals are shown

file 1: Table S5. SCC resolved malignant squamous cell cases from benign nodules (AUC $=0.653, \mathrm{p}=0.0258$ ). OPN, previously reported to be expressed at elevated levels in squamous cell carcinomas in tissue [39] and serum [40], also distinguished malignant squamous cell carcinoma cases from benign nodules (AUC $=0.664$, $p=0.0039$ ). The relatively strong performance of SCC and OPN in distinguishing squamous cell cases in the validation study is consistent with results observed in the training study (unpublished data).

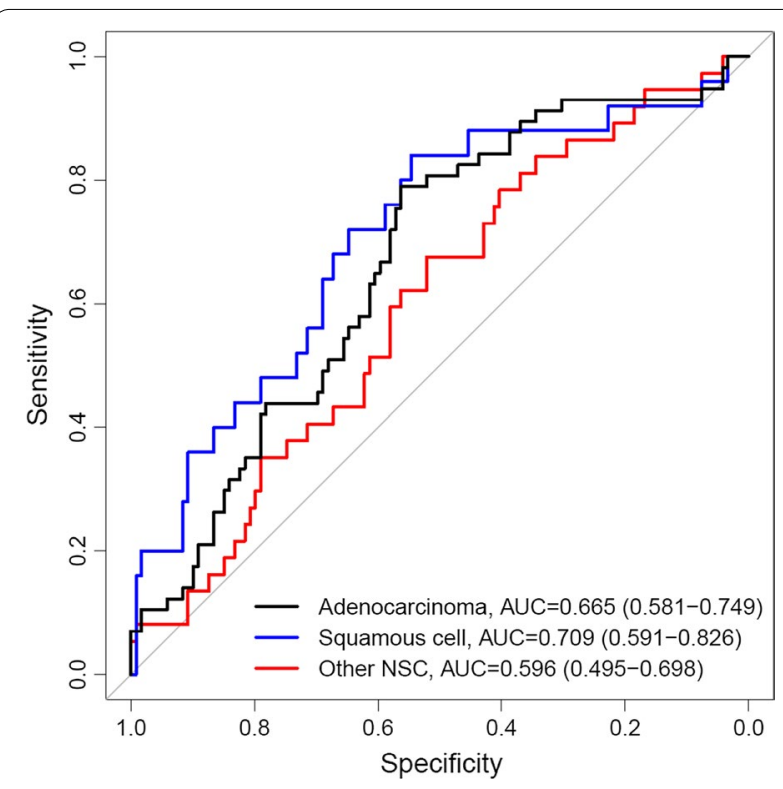

Fig. 3 ROC curves showing diagnostic accuracy of pulmonary nodule classifier (PNC) evaluated in PLCO nodule population $(n=119)$ relative to tumor histology: adenocarcinoma $(n=57$, black), squamous cell carcinoma ( $n=25$, blue), other non-small cell carcinomas $(n=37$, red). Area under the curve (AUC) and $95 \%$ confidence intervals are shown

As well as evaluating the diagnostic accuracy of the PNC in subjects with solitary pulmonary nodules, we also explored the performance of the classifier in individuals with other chest radiography findings considered suspicious for lung cancer (Table 2). While the PNC accurately distinguished patients with malignant masses (AUC $=0.718, \mathrm{p}<0.0001)$, it performed less well on subjects with other abnormal findings (infiltrate, hilar or pleural mass; AUC $=0.649, \mathrm{p}=0.0504$; Additional file 2: Figure S3). The performance of individual biomarkers, including those not selected for the PNC, was also assessed in these populations (Additional file 1: Table S6). CEA (AUC $=0.689 ; p=0.0004)$, and two of the biomarkers not included in the PNC, SPLI $($ AUC $=0.685$; $\mathrm{p}=0.0007)$ and TIMP1 (AUC $=0.670 ; \mathrm{p}=0.0019$ ), accurately resolved malignant masses from matched benign controls $(>3 \mathrm{~cm})$.

\section{Discussion}

The development of robust blood-based biomarkers to be used in the clinical setting requires thorough evaluation of pre-analytical variables, including the procedures used for blood collection and processing. Use of different blood collection tubes and variations in the time permitted for clot-formation can dramatically influence biomarker levels, potentially compromising the accuracy of test results $[26,41,42]$. In this preliminary evaluation 
of pre-analytical variability we examined the influence of clotting times on serum levels of candidate biomarkers in a limited number of health individuals $(n=6)$. Larger studies, including subjects with benign and malignant nodules are certainly warranted given the differential expression of circulating proteases in these populations [43]. Extending the time allowed for clot formation from $0.5 \mathrm{~h}$ to either $4 \mathrm{~h}$ or $24 \mathrm{~h}$ led to a substantial reduction in levels of MDK, prompting the removal of this biomarker from the panel of candidates. Additional studies investigating the influence or blood-collection tube-type and biomarker stability following serum separation revealed minimal levels of pre-analytical variability for the remaining 8 biomarkers candidates (unpublished data). These biomarkers were therefore included in the pool for multimarker development.

The diagnostic accuracy of the PNC fell dramatically in the validation study (AUC $=0.653$, Additional file 2: Figure S2) relative to the performance observed in the training study $(\mathrm{AUC}=0.897$, Additional file 2: Figure S1). Similar drops in multi-biomarker test performance have been reported for other tests in validation studies aimed at classifying indeterminate pulmonary nodules $[14,15]$. The decline in accuracy reflects diminished performance for all 5 biomarkers that constitute the PNC, with an average fall in AUC of 0.101 (Additional file 1: Table S7). Further investigation into differences in PNC signal revealed levels in the control populations (training and validation) to be remarkably similar (Additional file 2: Figure S4). This was not the case for the malignant populations, where PNC levels observed in the validation study were substantially lower than in the training study. Some of this difference may be attributed to the reduced dimensions of the malignant lesions included in the validation study. Subjects with malignant nodules (diameter $\leq 3 \mathrm{~cm}$ ) were selected for the validation study, while stage I NSCLC cases (diameter $\leq 5 \mathrm{~cm}$ ) were used for training. Although the reduced dimensions of malignant lesions are likely to have played some role in the lower PNC signal in validation study, the relatively low performance of the classifier in subjects with larger pulmonary masses (diameter $>3 \mathrm{~cm}$; $\mathrm{AUC}=0.718$, Additional file 2: Figure S3) suggests the involvement of additional factors. It seems likely that the pre-diagnostic collection of biospecimens, together with the asymptomatic nature of the population screened, may have impacted results in the validation study.

One of the key applications for an adjunctive test to be used in the management of individuals with indeterminate pulmonary nodules is to reduce the number of unnecessary biopsies in cases with low to moderate risk of malignancy, thereby reducing the number of biopsyrelated adverse events and the substantial costs associated with these procedures [10]. To achieve clinical utility in this setting, it has been suggested that an adjunctive test would need to perform with high accuracy: $80 \%$ sensitivity at $90 \%$ specificity [13]. At this level of performance, the test would reduce the number of unnecessary biopsies by approximately $80 \%$, making it a viable alternative to FDG-PET, which is typically recommended for evaluation of this population [7]. While FDG-PET typically performs with high accuracy in distinguishing benign from malignant nodules (sensitivity $=87 \%$, specificity $=83 \%$ ) [44], it is costly, and in some settings, including regions of endemic infectious granulomatous lung disease, it's use may be restricted [45]. With an AUC of 0.653 [95\% CI 0.583-0.723] (Additional file 2: Figure S2), the PNC classifier delivered $18 \%$ sensitivity at $90 \%$ specificity, well below of the desired level of performance. It remains to be seen whether integration of clinical and radiographic variables to the biomarkers included in the PNC will result in a multi-modal test with sufficient diagnostic accuracy to achieve clinical utility. Two of the biomarkers evaluated here (CEA, CYFRA 21-1) accurately distinguish malignant pulmonary lesions from benign nodules as individual markers (Table 3) and should be considered as possible components of future multi-marker panels.

Limitations of the study include the small number of biomarkers evaluated and differences in the clinical characteristics of the populations used in training and validation. Also lacking is a demonstration of clinical utility: does the PNC actually add to the accuracy of the clinical and radiographic variables typically assessed in patient evaluation? This type of multimodal analysis $[17,18,46-48]$ was not possible in the current study as some key radiographic variables, including nodule size, were not collected in the PLCO trial. A major strength of this study was the identification of candidate markers impacted by pre-analytical variables associated with sample collection and processing, and the deselection of such markers ahead of clinical validation studies. Another strength of the study stems from the utilization of samples collected in the PLCO trial to evaluate clinical accuracy. The PLCO study design not only overcomes any inherent bias in sample collection but also enables accurate selection of benign and malignant populations matched on radiographic findings and key demographic variables.

\section{Conclusions}

As CT-screening of the population at high risk for lung cancer gains traction, the need for non-invasive markers to improve clinical decision making in asymptomatic individuals with indeterminate nodules will become ever-more pressing. The PLCO study serves as a valuable 
resource in the testing of candidate biomarkers for the classification of benign nodules from malignant lesions in the setting of radiographic screening for lung cancer. Our study provides valuable insight into the clinical accuracy of a pulmonary nodule classifier and a number of well characterized biomarkers: CEA, CYFRA 21-1 and SCC, in the asymptomatic population. As well as evaluating diagnostic performance in individuals with pulmonary nodules, these biomarkers have also been characterized in subjects with other commonly encountered radiological findings. Some of the biomarkers included in the PNC should be further evaluated as viable complementing components of future blood-based adjunctive tests to be used in the diagnostic assessment of asymptomatic subjects with pulmonary nodules.

\section{Additional files}

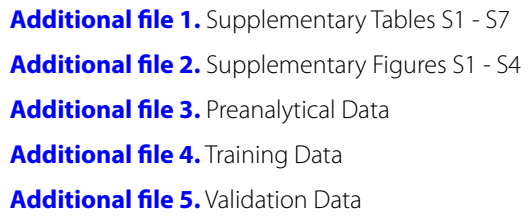

\begin{abstract}
Abbreviations
AUC: area under curve; CT: computed tomography; CEA: carcinoembryonic antigen-related cell adhesion molecule 5; CMS: Centers for Medicare \& Medicaid Services; ctDNA: circulating tumor DNA; CYFRA 21-1: cytokeratin-19 fragment; ELISA: enzyme-linked immunosorbent assay; FDG-PET: fluoro deoxy glucose-positron emission tomography; MDK: midkine; MMP2: matrix metalloproteinase-2; NLST: National Lung Screening Trial; NPV: negative predictive value; NSCLC: non-small cell lung cancer; OPN: osteopontin; PLCO: Prostate, Lung, Chest and Ovarian; PNC: pulmonary nodule classifier; PPV: positive predictive value; SCC: squamous cell carcinoma antigen; TFPI: tissue factor pathway inhibitor; TIMP1: tissue inhibitor of metalloproteinases-1; USPSTF: US Preventive Services Task Force.
\end{abstract}

\section{Authors' contributions}

CEB and RJL designed the study. HIP and WNR provided clinical samples and contributed to the study design. RJL provided statistical analysis. JLT provided immunoassay data. CEB wrote the manuscript with assistance from others. All authors read and approved the final manuscript.

\begin{abstract}
Author details
1 Quest Diagnostics, Research and Development, 33608 Ortega Highway, San Juan Capistrano, CA 92675, USA. ${ }^{2}$ Department of Cardiothoracic Surgery, NYU Langone Medical Center, 530 First Avenue, New York, NY 10016, USA. ${ }^{3}$ Division of Pulmonary, Critical Care, and Sleep Medicine, NYU School of Medicine, New York, NY 10016, USA. ${ }^{4}$ Present Address: Grifols Diagnostic Solutions, 4560 Horton St., Emeryville, CA 94608, USA.
\end{abstract}

\section{Acknowledgements}

We would like to thank Michael Furr, Craig Williams and John Commins (Information Management Services, Inc.) for their guidance in the design and execution of the validation study and for providing updated data from the PLCO cancer screening trial. We would also like to thank Claire S. Zhu and Paul F. Pinsky (Division of Cancer Prevention, NCl) for their initial analysis of the validation study data. We would also like to acknowledge Ted Cannon at Clinical Research Center at Cape Cod for his help in collection of biospecimens employed in pre-analytical studies and in model training.

\section{Competing interests}

CEB and RJL are currently employed by Quest Diagnostics. CEB is the inventor of lung cancer marker patents issued to Celera, a wholly-owned subsidiary of Quest Diagnostics.

\section{Availability of data and materials}

All data generated during this study are included in this published article. 3 xlsx files containing: preanalytical, training and validation datasets have been uploaded as additional files.

\section{Ethics}

Approval for clinical research protocols were provided by: Diagnostics IRB (CRCCC) and NYU School of Medicine IRB. Western IRB provided an exemption determination (\#1-728917-1) for samples collected in the PLCO study.

\section{Funding}

Support for biospecimen collection at NYU School of Medicine was provided by grants from the NCl Early Detection Research Network: U01CA086137 (WNR) and 2U01CA111295-04 (HIP).

\section{Publisher's Note}

Springer Nature remains neutral with regard to jurisdictional claims in published maps and institutional affiliations.

Received: 6 April 2017 Accepted: 10 June 2017

Published online: 05 July 2017

\section{References}

1. Gould MK, Tang T, Liu IL, Lee J, Zheng C, Danforth KN, Kosco AE, Di Fiore $J$, Suh DE. Recent trends in the identification of incidental pulmonary nodules. Am J Respir Crit Care Med. 2015;192:1208-14.

2. Moyer VA. USPSTF: screening for lung cancer: U.S. Preventive Services Task Force recommendation statement. Ann Intern Med. 2014;160:330-8.

3. CMS: National coverage determination (NCD) for screening for lung cancer with low dose computed tomography (LDCT). https://www.cms.gov/ newsroom/mediareleasedatabase/press-releases/2015-press-releasesitems/2015-02-05.html. 2015.

4. Aberle DR, Adams AM, Berg CD, Black WC, Clapp JD, Fagerstrom RM, Gareen IF, Gatsonis C, Marcus PM, Sicks JD. Reduced lung-cancer mortality with low-dose computed tomographic screening. N Engl J Med. 2011;365:395-409.

5. National Comprehensive Cancer Network. NCCN Clinical Practice Guidelines in Oncology. Lung Cancer Screening. Version 1. 2017_August 2016 www.nccn.org/professionals/physician_gls/pdf/lung_screening.pdf.

6. American College of Radiology. Lung CT screening reporting and data system (LUNG RADS ${ }^{\mathrm{TM}}$ ). https://www.acr.org/Quality-Safety/Resources/ LungRADS.

7. Gould MK, Donington J, Lynch WR, Mazzone PJ, Midthun DE, Naidich DP, Wiener RS. Evaluation of individuals with pulmonary nodules: when is it lung cancer? Diagnosis and management of lung cancer, 3rd ed: American College of Chest Physicians evidence-based clinical practice guidelines. Chest. 2013;143:e93S-120S.

8. Boiselle PM, Chiles C, Ravenel JG, White CS. Computed tomographic screening for lung cancer trends at leading academic medical centers from 2013 to 2015. JAMA Oncol. 2016;2:682-4.

9. Tanner NT, Aggarwal J, Gould MK, Kearney P, Diette G, Vachani A, Fang KC, Silvestri GA. Management of pulmonary nodules by community pulmonologists: a multicenter observational study. Chest. 2015;148:1405-14.

10. Lokhandwala T, Bittoni MA, Dann RA, D'Souza AO, Johnson M, Nagy RJ, Lanman RB, Merritt RE, Carbone DP. Costs of diagnostic assessment for lung cancer: a medicare claims analysis. Clin Lung Cancer. 2017;18:e27-34.

11. Ajona D, Pajares MJ, Corrales L, Perez-Gracia JL, Agorreta J, Lozano MD, Torre W, Massion PP, de-Torres JP, Jantus-Lewintre E, et al. Investigation of complement activation product $c 4 d$ as a diagnostic and prognostic biomarker for lung cancer. J Natl Cancer Inst. 2013;105:1385-93. 
12. Higgins G, Roper KM, Watson IJ, Blackhall FH, Rom WN, Pass HI, Ainscough $\mathrm{JF}$, Coverley D. Variant Ciz1 is a circulating biomarker for early-stage lung cancer. Proc Natl Acad Sci USA. 2012;109:E3128-35.

13. Bigbee WL, Gopalakrishnan V, Weissfeld JL, Wilson DO, Dacic S, Lokshin AE, Siegfried JM. A multiplexed serum biomarker immunoassay panel discriminates clinical lung cancer patients from high-risk individuals found to be cancer-free by CT screening. JThorac Oncol. 2012;7:698-708.

14. Daly S, Rinewalt D, Fhied C, Basu S, Mahon B, Liptay MJ, Hong E, Chmielewski G, Yoder MA, Shah PN, et al. Development and validation of a plasma biomarker panel for discerning clinical significance of indeterminate pulmonary nodules. J Thorac Oncol. 2013:8:31-6.

15. Li XJ, Hayward C, Fong PY, Dominguez M, Hunsucker SW, Lee LW, McLean M, Law S, Butler H, Schirm M, et al. A blood-based proteomic classifier for the molecular characterization of pulmonary nodules. Sci Transl Med. 2013;5:207ra142.

16. Mehan MR, Williams SA, Siegfried JM, Bigbee WL, Weissfeld JL, Wilson DO, Pass HI, Rom WN, Muley T, Meister M, et al. Validation of a blood protein signature for non-small cell lung cancer. Clin Proteomics. 2014;11:32.

17. Patz EF Jr, Campa MJ, Gottlin EB, Trotter PR, Herndon JE 2nd, Kafader D, Grant RP, Eisenberg M. Biomarkers to help guide management of patients with pulmonary nodules. Am J Respir Crit Care Med. 2013;188:461-5.

18. Pecot CV, Li M, Zhang XJ, Rajanbabu R, Calitri C, Bungum A, Jett JR, Putnam JB, Callaway-Lane C, Deppen S, et al. Added value of a serum proteomic signature in the diagnostic evaluation of lung nodules. Cancer Epidemiol Biomark Prev. 2012;21:786-92.

19. Kulpa J, Wojcik E, Reinfuss M, Kolodziejski L. Carcinoembryonic antigen, squamous cell carcinoma antigen, CYFRA 21-1, and neuron-specific enolase in squamous cell lung cancer patients. Clin Chem. 2002;48:1931-7.

20. Lai RS, Hsu HK, Lu JY, Ger LP, Lai NS. CYFRA 21-1 enzyme-linked immunosorbent assay. Evaluation as a tumor marker in non-small cell lung cancer. Chest. 1996;109:995-1000

21. Molina R, Filella X, Auge JM, Fuentes R, Bover I, Rifa J, Moreno V, Canals E, Vinolas N, Marquez A, et al. Tumor markers (CEA, CA 125, CYFRA 21-1, SCC and NSE) in patients with non-small cell lung cancer as an aid in histological diagnosis and prognosis. Comparison with the main clinical and pathological prognostic factors. Tumour Biol. 2003;24:209-18.

22. Pastor A, Menendez R, Cremades MJ, Pastor V, Llopis R, Aznar J. Diagnostic value of SCC, CEA and CYFRA 21.1 in lung cancer: a Bayesian analysis. Eur Respir J. 1997;10:603-9.

23. Birse CE, Lagier RJ, FitzHugh W, Pass HI, Rom WN, Edell ES, Bungum AO, Maldonado F, Jett JR, Mesri M, et al. Blood-based lung cancer biomarkers identified through proteomic discovery in cancer tissues, cell lines and conditioned medium. Clin Proteomics. 2015;12:18.

24. Jung YJ, Katilius E, Ostroff RM, Kim Y, Seok M, Lee S, Jang S, Kim WS, Choi CM. Development of a protein biomarker panel to detect non-small-cell lung cancer in Korea. Clin Lung Cancer. 2017:18:e99-107.

25. Fuzery AK, Levin J, Chan MM, Chan DW. Translation of proteomic biomarkers into FDA approved cancer diagnostics: issues and challenges. Clin Proteomics. 2013;10:13

26. Ostroff R, Foreman T, Keeney TR, Stratford S, Walker JJ, Zichi D. The stability of the circulating human proteome to variations in sample collection and handling procedures measured with an aptamer-based proteomics array. J Proteomics. 2010:73:649-66.

27. Timms JF, Arslan-Low E, Gentry-Maharaj A, Luo Z, T'Jampens D, Podust VN, Ford J, Fung ET, Gammerman A, Jacobs I, Menon U. Preanalytic influence of sample handling on SELDI-TOF serum protein profiles. Clin Chem. 2007:53:645-56.

28. Zhao X, Qureshi F, Eastman PS, Manning WC, Alexander C, Robinson WH, Hesterberg LK. Pre-analytical effects of blood sampling and handling in quantitative immunoassays for rheumatoid arthritis. J Immunol Methods. 2012;378:72-80

29. Prorok PC, Andriole GL, Bresalier RS, Buys SS, Chia D, Crawford ED, Fogel R, Gelmann EP, Gilbert F, Hasson MA, et al. Design of the prostate, lung, colorectal and ovarian (PLCO) cancer screening trial. Control Clin Trials. 2000;21:273S-3095.

30. Hayes RB, Reding D, Kopp W, Subar AF, Bhat N, Rothman N, Caporaso $\mathrm{N}$, Ziegler RG, Johnson CC, Weissfeld JL, et al. Etiologic and early marker studies in the prostate, lung, colorectal and ovarian (PLCO) cancer screening trial. Control Clin Trials. 2000;21:349S-55S.
31. Oken MM, Hocking WG, Kvale PA, Andriole GL, Buys SS, Church TR, Crawford ED, Fouad MN, Isaacs C, Reding DJ, et al. Screening by chest radiograph and lung cancer mortality: the Prostate, Lung, Colorectal, And Ovarian (PLCO) randomized trial. JAMA. 2011;306:1865-73.

32. Commins J, Furr M, Williams C. 2017. Current data from PLCO cancer screening trial was provided, including positive predictive values for individuals with a mass, nodule or other suspicious abnormality. Personal communication.

33. Hocking WG, Hu P, Oken MM, Winslow SD, Kvale PA, Prorok PC, Ragard LR, Commins J, Lynch DA, Andriole GL, et al. Lung cancer screening in the randomized Prostate, Lung, Colorectal, and Ovarian (PLCO) Cancer Screening Trial. J Natl Cancer Inst. 2010;102:722-31.

34. American Joint Committee on Cancer. AJCC Cancer Staging Manual. American Joint Committee on Cancer: Fifth Edition; 1997.

35. Hastie T, Zou H. Regularization and variable selection via the elastic net. JR Stat Soc. 2005:67:301-20

36. DeLong ER, DeLong DM, Clarke-Pearson DL. Comparing the areas under two or more correlated receiver operating characteristic curves: a nonparametric approach. Biometrics. 1988;44:837-45.

37. R Development Core Team: R: A language and environment for statistical computing. 2016. R Foundation for Statistical Computing, Vienna, Austria. ISBN 3-900051-07-0.

38. Tuck MK, Chan DW, Chia D, Godwin AK, Grizzle WE, Krueger KE, Rom W, Sanda M, Sorbara L, Stass S, et al. Standard operating procedures for serum and plasma collection: early detection research network consensus statement standard operating procedure integration working group. J Proteome Res. 2009;8:113-7.

39. Zhang J, Takahashi K, Takahashi F, Shimizu K, Ohshita F, Kameda Y, Maeda K, Nishio K, Fukuchi Y. Differential osteopontin expression in lung cancer. Cancer Lett. 2001;171:215-22.

40. Takenaka M, Hanagiri T, Shinohara S, Yasuda M, Chikaishi Y, Oka S, Shimokawa H, Nagata Y, Nakagawa M, Uramoto H, et al. Serum level of osteopontin as a prognostic factor in patients who underwent surgical resection for non-small-cell lung cancer. Clin Lung Cancer. 2013;14:288-94.

41. Banks RE, Stanley AJ, Cairns DA, Barrett JH, Clarke P, Thompson D, Selby PJ. Influences of blood sample processing on low-molecular-weight proteome identified by surface-enhanced laser desorption/ionization mass spectrometry. Clin Chem. 2005;51:1637-49.

42. Baumann S, Ceglarek U, Fiedler GM, Lembcke J, Leichtle A, Thiery J. Standardized approach to proteome profiling of human serum based on magnetic bead separation and matrix-assisted laser desorption/ionization time-of-flight mass spectrometry. Clin Chem. 2005;51:973-80.

43. Kerenidi T, Kazakou AP, Lada M, Tsilioni I, Daniil Z, Gourgoulianis KI. Clinical significance of circulating osteopontin levels in patients with lung cancer and correlation With VEGF and MMP-9. Cancer Invest. 2016:34:385-92.

44. Ost DE, Gould MK. Decision making in patients with pulmonary nodules. Am J Respir Crit Care Med. 2012;185:363-72.

45. Deppen S, Putnam JB Jr, Andrade G, Speroff T, Nesbitt JC, Lambright ES, Massion PP, Walker R, Grogan EL. Accuracy of FDG-PET to diagnose lung cancer in a region of endemic granulomatous disease. Ann Thorac Surg. 2011;92:428-33.

46. Dong J, Sun N, Li J, Liu Z, Zhang B, Chen Z, Gao Y, Zhou F, He J. Development and validation of clinical diagnostic models for the probability of malignancy in solitary pulmonary nodules. Thorac Cancer. 2014:5:162-8.

47. Vachani A, Pass HI, Rom WN, Midthun DE, Edell ES, Laviolette M, Li XJ, Fong PY, Hunsucker SW, Hayward C, et al. Validation of a multiprotein plasma classifier to identify benign lung nodules. J Thorac Oncol. 2015;10:629-37.

48. Yonemori K, Tateishi U, Uno H, Yonemori Y, Tsuta K, Takeuchi M, Matsuno Y, Fujiwara Y, Asamura H, Kusumoto M. Development and validation of diagnostic prediction model for solitary pulmonary nodules. Respirology. 2007;12:856-62. 